\title{
Mean heart dose-based normal tissue complication probability model for pericardial effusion
}

Junichi Fukada ( $\sim$ fukada@rad.med.keio.ac.jp )

Keio University

Kyohei Fukata

Keio University

Naoyoshi Koike

Keio University

Ryuichi Kota

Keio University

Naoyuki Shigematsu

Keio University

\section{Research Article}

Keywords: pericardial effusion, chemoradiotherapy, prediction

Posted Date: April 22nd, 2021

DOI: https://doi.org/10.21203/rs.3.rs-421111/v1

License: (c) (1) This work is licensed under a Creative Commons Attribution 4.0 International License.

Read Full License 
Mean heart dose-based normal tissue complication probability model for pericardial effusion

Junichi Fukada*a, Kyohei Fukata ${ }^{\mathrm{a}, \mathrm{b}}$, Naoyoshi Koike ${ }^{\mathrm{a}}$, Ryuichi Kota ${ }^{\mathrm{a}}$, Naoyuki Shigematsu ${ }^{\mathrm{a}}$

${ }^{a}$ Department of Radiology, School of Medicine, Keio University, School of Medicine, 35

Shinanomachi, Shinjuku-ku, Tokyo 160-8582, Japan

${ }^{b}$ Cancer Center, School of Medicine, Keio University, School of Medicine, 35 Shinanomachi,

Shinjuku-ku, Tokyo 160-8582, Japan

\section{*Corresponding author:}

Junichi Fukada

Department of Radiology, Keio University, School of Medicine, 35 Shinanomachi, Shinjuku-ku,

Tokyo 160-8582, Japan

Tel: $+81-3-3353-1211$

Fax: $+81-3-3359-7425$

E-mail: fukada@rad.med.keio.ac.jp

Sources of support: This work was supported by the JSPS KAKENHI Grant-in Aid for Early

Career Scientists given to N.K. (no. 20K16801) from the Ministry of Education, Culture, Sports,

Science, and Technology of Japan. 


\section{Abstract}

We investigated the normal tissue complication probability (NTCP) of the incidence of pericardial effusion (PCE) based on the mean heart dose (MHD). We reviewed medical records of oesophageal cancer patients treated with definitive chemoradiotherapy. Incidences of PCE in any grade (A-PCE) and symptomatic PCE (S-PCE) were evaluated separately. To identify significant predictors for PCE, clinical and dose-volume parameters were analysed using a receiver operating characteristic (ROC) curve and multivariate regression analysis. The generated NTCP model was compared to the Lyman-Kutcher-Burman (LKB) model to validate its clinical applicability. Among 229 eligible patients, A-PCE and S-PCE were observed in 100 (43.7\%) and $18(7.9 \%)$ patients, respectively. MHD showed a preferable area under the curve (AUC) value for S-PCE (AUC=0.821) and A-PCE (AUC=0.734). MHD was the only significant predictor for A-PCE; MHD and hypertension were selected as significant factors for S-PCE. The estimated NTCP, using the MHD-based model, showed excellent correspondence to the LKB model in A-PCE and S-PCE. The NTCP curve of A-PCE was gentler than that of SPCE and had no threshold. The MHD-based NTCP model was simple but comparable to the LKB model for both A-PCE and S-PCE. Estimated NTCP may provide clinically useful parameters for predicting PCE. 


\section{Introduction}

Radiation therapy plays an important role in cancer treatment; however, cardiac toxicities are among the late adverse events associated with thoracic irradiation [1-11]. Partial volume radiation-induced heart complications mostly occur in long-surviving breast cancer patients who have undergone surgery [1,2]; meanwhile, evidence on radiation-related injuries after whole heart radiation has come mostly from patients with Hodgkin lymphoma [3]. Recently, cardiotoxicity has been recognised as an adverse event that occurs earlier than previously thought, as indicated by studies on patients with lung and oesophageal cancer [4-8]. Furthermore, some reports have indicated that heart irradiation might affect survival even in the short-term period [6-8]. Among the various cardiac complications such as pericarditis, pericardial effusion (PCE), myocardial infarction, angina, and arrhythmia, PCE is regarded the most frequent and important toxicity $[2,4,8-11]$. Although it has been suggested that reduced irradiation dose and volume delivered to the heart may help manage the risk of complications, the quantitative evaluation of the risk for dose-volume irradiation to the heart remains unclear. It is reasonable to introduce mathematical modelling to analyse the radiation-to-heart dose-volume relationship and predict the normal tissue complication probability (NTCP).

We have previously reported the incidence of symptomatic PCE (S-PCE) in oesophageal cancer patients, showing that the average irradiation dose to the heart (including 
the pericardium) was the most significant predictor of S-PCE [11]. As the pericardium appears to be an anatomically uniform structure, we have assumed it to be a parallel organ. This study aimed to investigate the NTCP of PCE using mathematical modelling and examine whether the mean heart dose (MHD) can be used as an indicator of NTCP in S-PCE and PCE of any grade (A-PCE). 


\section{Methods}

The patient dataset analysed in this study was acquired from radiation treatment records of The Department of Radiation Oncology, Keio University Hospital. This was a retrospective observational study; hence, we used the "opt-out" method to obtain informed consent from patients. The study was approved by Independent Ethics Committee, Keio University School of Medicine (no. 20150137). Patient clinical information was anonymised to protect personal information. All investigations were conducted in accordance with the relevant guidelines and regulations.

Patients were eligible for inclusion in the present study if they met the following criteria: newly diagnosed primary oesophageal cancer patients who received definitive concurrent chemoradiotherapy (CCR) between 2001 and 2014, involvement of the thoracic oesophagus, treated with conventional 2.0 or 1.8 Gy fractionation, total irradiation dose of $\geq 50$ Gy, computed tomography (CT) data were available for analysis of the dose-volume of the pericardium, $\geq 6$ months follow-up, and no evidence of malignant PCE. Two-dimensional treatment plans were reconstructed as three-dimensional plans using a treatment planning system without modification, the details of which have been previously reported [11].

Follow-up chest-abdominal CT scans and gastrointestinal fibrescopy were performed every 3-6 months for 3 years, and half-yearly to annual examinations were performed after 3 
years. Adverse events were graded according to the National Cancer Institute-Common Terminology Criteria for Adverse Events (NCI-CTCAE) version 4.0 [12]. S-PCE was defined as an effusion of grade 3 or above. The survival and PCE- free periods were determined after the completion of CCR. Heart volume is defined as the volume enclosed by the pericardium including the heart [11]. In this study, however, the pericardial volume was defined separately as a ring structure consisting of $2 \mathrm{~mm}$ of both outer and inner walls of the heart. The cardiac substructures, including the right and left atria and the right and left ventricles, were also manually contoured on each CT image, referring to the Atlases for Organs at Risk (OARs) in Thoracic Radiation Therapy [13] and the heart atlas by Feng et al. [14]. All contours were reviewed and corrected by a single radiation oncologist (J.F.). Three-dimensional plans were generated in increments of $10 \mathrm{cGy}$, using a commercially available radiation treatment planning system $\left(\mathrm{XiO}^{\circledR}\right.$, Elekta $\mathrm{AB}$, Stockholm, Sweden), and calculated with the superposition algorithm appropriate for use with heterogeneous tissues. Calculated dose distribution was acquired by exporting the Digital Imaging and Communications in Medicine in radiation therapy (DICOM RT) file.

\section{Statistical calculation}

Univariate logistic regression analysis was calculated with a cut-off $\mathrm{p}$-value $<0.10$ to analyse the relationship between PCE and patient and treatment characteristics. Pearson 
correlations were used to test multicollinearity with an R-squared threshold $>0.70$. A

multivariable forward stepwise logistic regression model was fit to include all selected

significant factors associated with PCE. Variable parameters were included in the final model

once the model was found to be significantly better using the likelihood ratio test ( $p<0.05$, twosided).

To validate our model internally, all patient and treatment factors and selected

dosimetric parameters for the cause of the toxicities were repeated in 1000 bootstrap samples.

The optimism of the generated model was assessed by estimating the performance difference between each bootstrap and the original sample in line with the Transparent Reporting of a multivariable prediction model for Individual Prognosis or Diagnosis (TRIPOD) statement [15]. The area and the adjusted area under the receiver operating characteristic (ROC) curve were compared to quantitatively evaluate the predictive power of the analyses. To perform an external validation, a previously reported model [16] was compared to our models.

To compare and validate the MHD-based NTCP model, we used probit regression and compared it to the Lyman-Kutcher-Burman (LKB) NTCP model [17]. Generalised equivalent uniform dose (gEUD)-based LKB NTCP parameters (n, m, and $\mathrm{TD}_{50}$ ) were estimated using the maximum likelihood method [18]. In the probit regression-using MHD-based NTCP model, the $\mathrm{m}$-value and $\mathrm{TD}_{50}$ were estimated given $\mathrm{n}=1$, with the pericardium considered as an organ with 
a large volume effect. In this case, $\mathrm{TD}_{50}$ represented $\mathrm{MHD}$, instead of gEUD.

NTCP parameter optimisation from the DICOM RT file was performed using an in-

house application. The application was coded with Python, Pydicom, dicompyler-core, and

scikit-learn [19-22]. Optimum parameters were determined using the gradient descent method.

The probability of pericarditis incidence was given by

$$
\mathrm{NTCP}=\frac{1}{\sqrt{2 \pi}} \int_{-\infty}^{t} \exp \left(-\frac{x^{2}}{2}\right) d x
$$

with

$$
\begin{gathered}
t=\frac{\operatorname{gEUD}-\mathrm{TD}_{50}}{m \cdot \mathrm{TD}_{50}} \\
\text { gEUD }=\left(\sum_{i} v_{i} D_{i}^{\frac{1}{n}}\right)^{n}
\end{gathered}
$$

where $\mathrm{n}, \mathrm{m}$, and $\mathrm{TD}_{50}$ are the parameters of the $\mathrm{LKB}$ model, including the dose-volume effect

on the organ; m-value represents the steepness of the dose-response curve, n-value represents

the volume effect (large volume effect for $\mathrm{n}$ close to one; small volume effect for $\mathrm{n}$ close to

zero), and $\mathrm{TD}_{50}$ represents the dose that corresponds to the $50 \%$ probability of complications,

given whole-organ irradiation. In the third equation, $\mathrm{v}_{\mathrm{i}}$ and $\mathrm{D}_{\mathrm{i}}$ denote fractional volume and dose per ith voxel, respectively.

The following equation was used to calculate the coefficient of determination:

$$
R^{2}=1-\sum_{i}\left(y_{i}-f\right)^{2} / \sum_{i}\left(y_{i}-\bar{y}\right)^{2}
$$


Herein, $\mathrm{f}$ denotes the linear function calculated by regression and $\bar{y}$ is the arithmetic mean of a vertical axis in the figure.

Statistical analyses were performed in SPSS, version 26.0 (SPSS Inc. Chicago Illinois, USA). 


\section{Results}

Patient background and incidence of pericardial effusion

A total of 229 consecutive patients with oesophageal cancer met our inclusion criteria.

The overall median follow-up period was 37 months (range, 6-183), and that for surviving patients (n=131) was 48 months (range, 6-178). A-PCE was observed in $100(43.7 \%)$ patients.

The timing of PCE onset ranged from 2-75 months, with a median of 7 months. S-PCE

developed in 18 (7.9\%) patients; the timing of onset was 4-108 months, with a median of 21

months. Other observed new cardiac events were coronary artery disease $(n=8)$, arrhythmia $(n=6)$, and heart failure $(n=7)$. Patient demographic and clinical characteristics are summarised in Table 1.

Significant predictor identification for PCE by logistic regression analysis

Univariable logistic regression analysis demonstrated that most cardiopulmonary dose parameters were significantly associated with both A-PCE and S-PCE (suppl. data Table 1 and 2). Of the clinical factors, the use of alcohol was associated with A-PCE and age, hypertension, and performance status were associated with S-PCE. Most cardiac dose-volume parameters were highly correlated in predicting A-PCE and S-PCE by Pearson's correlation analysis. Then we performed a ROC analysis of the cardiopulmonary dose-volume parameters to evaluate the relevance of PCE (suppl. data Table 3 and 4). MHD was selected as the significant predictor 
with the largest area under the curve (AUC) value for S-PCE (AUC=0.821) by ROC analysis. While for A-PCE, the AUC of MHD (AUC=0.713) was smaller than that of heart V40 (AUC=0.734), we thought MHD can be regarded as a representative parameter considering its clinical usefulness and preferable AUC values (suppl. data Table 5 and 6). Multivariable logistic regression analysis demonstrated that only the MHD was the best predictor for A-PCE, with an odds ratio of 1.08 per Gy MHD (adjusted AUC after bootstrapping: 0.715). S-PCE was best predicted by MHD and hypertension, with an odds ratio of 1.17 (adjusted AUC after bootstrapping $=0.814)$. The estimated model and NTCP curve by logistic regression are presented in Table 2a and Fig. 1, respectively. The robustness of choosing MHD in the NTCP model, both for A-PCE (suppl. data Fig. 1 and 2) and S-PCE (suppl. data Fig. 3 and 4), was verified by bootstrap analysis and calibration. The results of the previously reported NTCP model [16] are also shown in Table $2 \mathrm{a}$ as an external validation, indicating comparable results of discrimination (AUC) and calibration.

NTCP model by probit regression analysis

The NTCP curve for A-PCE derived from the MHD-based model calculated by probit regression and derived from the LKB model are shown in Fig. 2. The NTCP curve derived from the MHD-based model (Fig. 2a) showed a gentler curve $(m=0.75)$ than that derived from the LKB model (Fig. 2b) (m=0.47); moreover, the NTCP curve derived from the MHD-based 
model showed preferable fitting by linear regression (Fig. 2c). The NTCP increased by $1.3 \%$ for each $1.0 \mathrm{~Gy}$ mean dose increase; the intercept was $5.1 \%$ at $0 \mathrm{~Gy}$. When validating the goodness of fit, both the discrimination and the calibration of A-PCE in the MHD-based model were equivalent to that in the LKB model (Table $2 \mathrm{~b}$ and suppl. data Fig. 5). On the other hand, the NTCP model for S-PCE derived from the MHD-based model calculated by probit regression and derived from the LKB model are shown in Fig. 3. The MHD-based model (Fig. 3a) was of similar shape and yielded similar estimates (Table 2b) as the LKB model (Fig. 3b). Regarding the validation of the goodness of fit, discrimination and the calibration of S-PCE in the MHDbased model were equivalent to that in the LKB model (Table 2b and suppl. data Fig. 6). As a direct comparison of the MHD and LKB models, the results of linear regression analysis and calibration indicated excellent correspondence in both A-PCE and S-PCE (Table 2b, suppl. data Fig. 7 and 8$)$.

Assessment of NTCP estimates

NTCP estimates derived from the logistic and probit regression are summarised in Table 3. The NTCP model for S-PCE showed a similar shape at the threshold near 30 Gy (Fig. 1b, 3a, 3b); however, the NTCP model for A-PCE, while also showing a similar shape, showed a curve that was gentler than that of S-PCE without threshold (Fig. 1a, 2a, 2b). 


\section{Discussion}

In this study, we investigated the NTCP of PCE using mathematical modelling. MHD was identified as the most significant predictor for S-PCE and also as a relevant parameter for A-PCE in multivariate analysis. The bootstrap analysis confirmed the robustness of obtaining a preferable AUC by selecting MHD in the NTCP model. The NTCP model derived from MHD was comparable to the widely used LKB model for both A-PCE and S-PCE.

Several previous studies investigated clinical background as a predictor for PCE [4, $10,11,16]$. The lung disease study [10] included receipt of adjuvant chemotherapy, history of cardiac disease, and presence of a left-sided tumour. In the current study, hypertension was selected as the only clinical factor only for the S-PCE. A history of hypertension may induce cardiac symptoms in patients with A-PCE. Further investigation is needed to elucidate the mechanisms since PCE arises from various processes and factors (cardiogenic, infectious, malignant etc.).

In this study, various dose-volume parameters such as heart, pericardium, atria, and ventricles were widely selected as significant factors. These heart and cardiac substructures are highly correlated; therefore, we selected MHD as a representative of cardiac dose-volume parameters considering the results of ROC and the widely used guidelines [23]. Several previous studies have reported that the dose-volume of the irradiated pericardium may be a better factor 
than the dose volume of the whole heart $[4,16]$, and the bootstrap analysis of our study suggests the same. Since the dose volume of the whole heart was equivalent to that of the pericardium, we applied MHD to the NTCP model after considering its simplicity in clinical application. Some previous studies have investigated NTCP of cardiac toxicities in oesophageal cancer patients. According to studies by Emami and Burman et al., which proposed the LKB model, $\mathrm{TD}_{50}$ for the development of pericarditis was $48 \mathrm{~Gy}$, with $\mathrm{n}$ - and m-value of 0.35 and 0.10 , respectively $[17,24]$. In the present study, the estimated $\mathrm{TD}_{50}$ and $\mathrm{n}$-values corresponding to $\mathrm{S}$ PCE onset were nearly equivalent to those reported previously $[17,24]$. The dose-volume associated with the development of pericarditis may correspond to that associated with the development of S-PCE. Meanwhile, several studies had investigated NTCP for A-PCE. Martel et al. reported an n-value of 0.63 for PCE, which may indicate that the pericardium is a parallel organ. These authors evaluated $\mathrm{TD}_{50}$ as a 'biodose', based on the data from a prospective clinical trial using a hypofractionation schedule, showing that $\mathrm{TD}_{50}$ was $50.6 \mathrm{~Gy}$ and $\mathrm{m}$-value was 0.13 , both of which are estimates comparable to those reported in the present study [25]. Recently, Beukema et al reported NTCP for A-PCE using pericardial mean dose by logistic analysis [16]. Results of our external validation were per the TRIPOD statement, as the discrimination ability and calibration were comparable to their results. In this study, the NTCP curve for A-PCE showed a gentler shape than S-PCE without threshold. A-PCE showed 
preferable fitting by a straight line with intercept, which may indicate that the risk of A-PCE increases linearly with MHD. A previous report on the development of coronary artery disease in breast cancer patients indicated that disease risk increased linearly with the administration of MHD [26]. Similarly, another breast cancer study reported a predication model for acute cardiac events that concluded that LV-V5 (left ventricular volume receiving $5 \mathrm{~Gy}$ ) was the predictor [27]. These results may suggest that even low doses cause cardiotoxicity.

Some previous studies have assumed $\mathrm{n}$ to equal one when considering other organs at risk to be parallel organs; for example, lungs, salivary glands, and liver [28-30]. A previous study of radiation pneumonitis by Semenenko and Li compared NTCP estimates of cases with steroid administration with those of all cases showing that the NTCP sigmoid curve for all events followed a gentler progression with a lower threshold of mean total lung dose than that for cases of severe pneumonitis that required treatment with steroids [29]. These findings correspond to the present findings on NTCP curves for A-PCE and S-PCE.

This study has some limitations, which should be considered when interpreting its findings. First, as this was a retrospective single-centre study, the sample size and the number of symptomatic cardiac events except for PCE was small. Hence, the estimated NTCP was limited to PCE, not to whole cardiac events. Second, the estimated dose volume of cases treated with a two-dimensional plan might be inaccurate due to reconstruction. Since S-PCE was observed 
relatively frequently in the early period, we included these initial cases in the NTCP analysis.

Finally, mathematical models have inherent limitations, as they do not consider host factors.

These limitations notwithstanding, the present study has several strengths. A

substantial number of cases were treated using conventional fractionated irradiation and cardiac and lung structures were delineated by methods that have been previously reported as atlases and are now included among the standard. We clarify the difference in the NTCP curve for APCE and S-PCE. This model is simpler than the LKB model and can be used in the clinical context. Although the reported n-value was consistent with that shown in a previous study, we could not demonstrate that the pericardium was a parallel organ. Further studies may elucidate the impact of irradiation on a specific cardiac substructure encompassed by the pericardium.

\section{Conclusions}

The MHD was identified as a significant predictor for PCE risk assessment with preferable ROC and robustness. The MHD-based NTCP model is simple but comparable to the LKB model for both A-PCE and S-PCE. Estimated NTCP provides clinically useful parameters for predicting PCE. Hypertension may enhance symptoms in the presence of PCE.

\section{References}


1. Darby, S. et al. Mortality from cardiovascular disease more than 10 years after radiotherapy for breast cancer: nationwide cohort study of 90000 Swedish women. BMJ. 326, 256-257 (2003).

2. Demirci, S., Nam, J., Hubbs, J. L., Nguyen, T. \& Marks, L. B. Radiation-induced cardiac toxicity after therapy for breast cancer: interaction between treatment era and follow-up duration. Int. J. Radiat. Oncol. Biol. Phys. 73, 980-987 (2009).

3. Hancock, S. L., Donaldson, S. S. \& Hoppe, R. T. Cardiac disease following treatment of Hodgkin's disease in children and adolescents. J. Clin. Oncol. 11, 12080-12115 (1993).

4. Wei, X. et al. Risk factors for pericardial effusion in inoperable esophageal cancer patients treated with definitive chemoradiation therapy. Int. J. Radiat. Oncol. Biol. Phys. 70, 707-714 (2008).

5. Kato, K. et al. Phase II study of chemoradiotherapy with 5-fluorouracil and cisplatin for Stage II-III esophageal squamous cell carcinoma: JCOG trial (JCOG 9906). Int. J. Radiat. Oncol. Biol. Phys. 81, 684-690 (2011).

6. Bradley, J. D. et al. Standard-dose versus high-dose conformal radiotherapy with concurrent and consolidation carboplatin plus paclitaxel with or without cetuximab for patients with stage IIIA or IIIB non-small-cell lung cancer (RTOG 0617): a randomised, two-by-two factorial phase 3 study. Lancet. Oncol. 16, 187-199 (2015). 
7. Contreras, J. A. et al. Cardiac dose is associated with immunosuppression and poor survival in locally advanced non-small cell lung cancer. Radiother. Oncol.128, 498-504 (2018).

8. Wang, K. et al. Cardiac toxicity after radiotherapy for stage III non-small-cell lung cancer: pooled analysis of dose-escalation trials delivering 70 to 90 Gy. J. Clin. Oncol. 35, 1387-1394 (2017).

9. Adams, M. J., Hardenbergh, P. H., Constine, L. S. \& Lipshultz, S. E. Radiationassociated cardiovascular disease. Crit. Rev. Oncol. Hematol. 45, 55-75 (2003).

10. Ning, M. S. et al. Incidence and predictors of pericardial effusion after chemoradiation therapy for locally advanced non-small cell lung cancer. Int. J. Radiat. Oncol. Biol. Phys. 99, 70-79 (2017).

11. Fukada, J. et al. Symptomatic pericardial effusion after chemoradiation therapy in esophageal cancer patients. Int. J. Radiat. Oncol. Biol. Phys. 87, 487-493 (2013).

12. National Cancer Institute (NCI). Cancer Therapy Evaluation Program (CTEP), http://ctep.cancer.gov/protocolDevelopment/electronic applications/ctc.htm\#ctc 40; $\underline{2021}$ [accessed 13 January 2021]. 
13. Kong, F. M., Quint, L., Machtay, M. \& Bradley, J. Atlases for Organs at Risk (OARs) in Thoracic Radiation Therapy; RTOG, https://www.nrgoncology.org/ciro-lung; 2021 [accessed 13 January 2021].

14. Feng, M. et al. Development and validation of a heart atlas to study cardiac exposure to radiation following treatment for breast cancer. Int. J. Radiat. Oncol. Biol. Phys.79, 10$18(2011)$.

15. Moons, K. G. et al. Transparent reporting of a multivariable prediction model for Individual Prognosis or Diagnosis (TRIPOD): explanation and elaboration. Ann. Intern. Med.162, W1-73 (2015).

16. Beukema, J. C. et al. Can we safely reduce the radiation dose to the heart while compromising the dose to the lungs in oesophageal cancer patients? Radiother. Oncol.149, 222-227 (2020).

17. Burman, C., Kutcher, G. J., Emami, B. \& Goitein, M. Fitting of normal tissue tolerance data to an analytic function. Int. J. Radiat. Oncol. Biol. Phys. 21, 123-135 (1991).

18. Niemierko, A. Reporting and analyzing dose distributions: a concept of equivalent uniform dose. Med. Phys. 24, 103-110 (1997). 
19. Rossum, G. Python reference manual. CWI (Centre for Mathematics and Computer Science), https://dl.acm.org/doi/book/10.5555/869369; 1995 [accessed 13 January 2021].

20. Darcy Mason, scaramallion, rhaxton et al. pydicom/ pydicom: v1.4.0, https://github.com/pydicom/pydicom/releases/tag/v1.4.0;2020 [accessed 13 January 2021].

21. Aditya Panchal, pyup.io bot, Gabriel Couture et al. Dicompyler/Dicompyler-core v0.5.5, https://github.com/dicompyler/dicompyler-core/tree/v0.5.5; 2019 [accessed 13 January 2021].

22. Pedregosa, F. et al. Scikit-learn: Machine learning in Python. JMLR. 12, 2825-2830 (2011).

23. NCCN Clinical Practice Guidelines in Oncology Non-Small Cell Lung Cancer Version 3, https://www.nccn.org/professionals/physician_gls/pdf/nscl.pdf, 2020 [accessed 2 November 2020].

24. Emami, B. et al. Tolerance of normal tissue to therapeutic irradiation. Int. J. Radiat. Oncol. Biol. Phys. 21, 109-122 (1991). 
25. Martel, M. K., Sahijdak, W. M., Ten Haken, R. K., Kessler, M. L., \& Turrisi, A. T.

Fraction size and dose parameters related to the incidence of pericardial effusions. Int.

J. Radiat. Oncol. Biol. Phys. 40, 155-161 (1998).

26. Darby, S. C. et al. Risk of ischemic heart disease in women after radiotherapy for breast cancer. N. Engl. J. Med. 368, 987-998 (2013).

27. van den Bogaard, V. A. et al. Validation and modification of a prediction model for acute cardiac events in patients with breast cancer treated with radiotherapy based on three-dimensional dose distributions to cardiac substructures. J. Clin. Oncol. 35, 11711178 (2017).

28. Kwa, S. L. et al. Radiation pneumonitis as a function of mean lung dose: an analysis of pooled data of 540 patients. Int. J. Radiat. Oncol. Biol. Phys. 42, 1-9 (1998).

29. Semenenko, V. A. \& Li, X. A. Lyman-Kutcher-Burman NTCP model parameters for radiation pneumonitis and xerostomia based on combined analysis of published clinical data. Phys. Med. Biol. 53, 737-755 (2008).

30. Dawson, L. A. et al. Analysis of radiation-induced liver disease using the Lyman NTCP model. Int. J. Radiat. Oncol. Biol. Phys. 53, 810-821 (2002). 


\section{Author contributions}

Conception and design of the work: J.F., Data acquisition N.K., R.K. Data analysis and

interpretation: J.F., K.F. Writing, revision of the manuscript: J.F. Study supervision: N.S. All

authors have read and approved the final version of the manuscript and agree with the order of presentation of the authors.

Conflicts of interest: The authors declare no conflict of interest. 


\section{Figure legends}

Figure 1. NTCP curves for A-PCE (a) and S-PCE (b).

The NTCP curves were calculated by the logistic regression analysis derived from MHD.

A-PCE, pericardial effusion in any grade; NTCP, normal tissue complicated probability; S-PCE, symptomatic pericardial effusion; MHD, mean heart dose

Figure 2. NTCP curves for A-PCE calculated by the MHD-based model (a and c) and LKB model (b).

The x-axis indicates the MHD, which corresponds to the mean dose in the MHD-based model.

Filled circles indicate average dose points for gEUD at 10 Gy intervals. NTCP curve by the MHD-based model also showed preferable fitting by linear regression (c).

A-PCE, pericardial effusion in any grade; LKB model, Lyman-Kutcher-Burman model; MHD, mean heart dose; NTCP, normal tissue complicated probability; No. of Pts, number of patients; gEUD, generalised equivalent uniform dose

Figure 3. NTCP curves for S-PCE calculated by the (a) MHD-based model and (b) LKB model.

The x-axis indicates gEUD, which corresponds to the mean dose in the MHD-based model.

Filled circles indicate average dose points for gEUD at 10 Gy intervals. 
gEUD, generalised equivalent uniform dose; LKB model, Lyman-Kutcher-Burman model; No.

of Pts, number of patients; NTCP, normal tissue complicated probability; S-PCE, symptomatic

pericardial effusion; MHD, mean heart dose

\section{TABLES}

Table 1. Demographic and clinical characteristics of patients included in the study cohort

\begin{tabular}{lll}
\hline Characteristic & $\mathrm{n}=229 \quad(\%)$ \\
\hline
\end{tabular}

Age (years)

median [range]

$67 \quad[43-87]$

Sex

$\begin{array}{crc}\text { Male } & 196 & (85.6) \\ \text { Female } & 33 & (14.4)\end{array}$

\section{WHO Performance status}

$\begin{array}{lrl}\mathbf{0} & 69 & (30.1) \\ \mathbf{1} & 134 & (58.5) \\ \mathbf{2} & 26 & (11.4)\end{array}$

Hypertension

$\begin{array}{lrr}\text { yes } & 69 & (30.1) \\ \text { no } & 160 & (69.9)\end{array}$

Smoking history

$\begin{array}{crl}\text { yes } & 55 & (24.0) \\ \text { no } & 171 & (74.7) \\ \text { unknown } & 3 & (1.3)\end{array}$

Use of alcohol

$\begin{array}{lrr}\text { yes } & 27 & (11.8) \\ \text { no } & 196 & (85.6)\end{array}$


unknown

Diabetes mellitus

yes

Cardiovascular disease

$\begin{array}{lrr}\text { yes } & 28 & (12.2) \\ \text { no } & 201 & (87.8)\end{array}$

Main tumour location

$\begin{array}{rrr}\text { Ut } & 55 & (24.0) \\ \text { Mt } & 114 & (49.8) \\ \text { Lt } & 60 & (26.2)\end{array}$

Clinical stage

$\begin{array}{lll}\text { I } & 78 & (34.1) \\ \text { II } & 43 & (18.8) \\ \text { III } & 83 & (36.2) \\ \text { IV } & 25 & (10.9)\end{array}$

Heart volume (mL) median [range] $715 \quad 361-1188$

Radiation dose (Gy)

$\begin{array}{rrr}60 & 146 & (63.8) \\ <60 & 83 & (36.2)\end{array}$

Treatment planning

$\begin{array}{lrr}\text { 2D-plan } & 83 & (36.2) \\ \text { 3D-CRT } & 146 & (63.8)\end{array}$

CCR regimen

$\begin{array}{crc}\text { CDDP + 5-FU } & 210 & (91.7) \\ \text { 5-FU } & 3 & (1.3) \\ \text { CDDP + TS-1 } & 9 & (3.9) \\ \text { Docetaxel } & 7 & (3.1)\end{array}$

MHD (Gy)

median [range] 
2D-plan: two-dimensional treatment plan; 3D-CRT: three-dimensional conformal radiotherapy;

5-FU: 5-fluorouracil; CCR: concurrent chemoradiotherapy; CDDP: cisplatin; Lt: lower thoracic oesophagus; MHD: mean heart dose; Mt: middle thoracic oesophagus; Ut: upper thoracic oesophagus. 
Table 2. NTCP models for A-PCE and S-PCE by logistic regression analyses (2a) and by probit regression analyses (2b)

(Table 2a)

Predictor Coefficient Constant Odds ratio CI (95\%) Significance Discrimination Adjusted Hosmer-

(AUC) AUC Lemeshow

\begin{tabular}{llllllllll}
\multicolumn{10}{c}{ (Chi-Square, p) } \\
\hline A-PCE & MHD & 0.073 & -2.513 & 1.076 & $1.05-1.11$ & 0.000 & $0.713[0.65-0.78]$ & 0.715 & $13.05,0.22$ \\
A-PCE* & MPD & & & 1.11 & $1.06-1.16$ & 0.00 & $0.73[0.66-0.80]$ & 0.70 & $2.86,0.94$ \\
S-PCE & MHD & 0.161 & -7.240 & 1.174 & $1.09-1.27$ & 0.000 & $0.821[0.73-0.91]$ & 0.814 & $10.69,0.36$ \\
& HTN & -1.858 & & 0.156 & $0.047-0.51$ & 0.002 & & &
\end{tabular}

\begin{tabular}{lllllllll} 
(Table 2b) & & & & & & \\
\hline & model & n & m & TD50 & log-likelihood & AUC & $\begin{array}{l}\text { Hosmer-Lemeshow } \\
\text { (Chi-Square, p) }\end{array}$ & $\begin{array}{l}\text { coefficient of } \\
\text { determination }\end{array}$ \\
\hline A-PCE & LKB & 0.38 & 0.47 & 41.0 & -140.90 & $0.718[0.65-0.79]$ & $8.55,0.382$ & 0.95 \\
& MHD-based & 1 & 0.75 & 34.3 & -141.92 & $0.701[0.63-0.77]$ & $8.15,0.418$ & \\
S-PCE & LKB & 0.36 & 0.19 & 57.7 & -52.39 & $0.802[0.68-0.93]$ & $5.41,0.713$ & 0.95 \\
& MHD-based & 1 & 0.26 & 56.5 & -51.94 & $0.809[0.69-0.93]$ & $8.64,0.373$ & \\
\hline
\end{tabular}

*A-PCE indicates pericardial effusion in any grade according to the NTCP model previously reported [16].

A-PCE: pericardial effusion in any grade; AUC: area under the curve; CI (95\%): 95\% confidence interval; LKB: Lyman-Kutcher-Burman; MHD:

mean heart dose; MPD: mean pericardial dose; NTCP: normal tissue complication probability; S-PCE: symptomatic pericardial effusion. 
Table 3. Summary of the estimated TD and normal tissue complication probability stratified to the applied model and the grade of pericardial effusion

\begin{tabular}{|c|c|c|c|c|c|c|}
\hline & \multicolumn{2}{|c|}{$\begin{array}{c}\text { MHD-based } \\
\text { (logistic) }\end{array}$} & \multicolumn{2}{|c|}{$\begin{array}{c}\text { MHD-based } \\
\text { (probit) }\end{array}$} & \multicolumn{2}{|c|}{$\begin{array}{c}\text { LKB } \\
\text { (probit) }\end{array}$} \\
\hline & A-PCE & S-PCE & A-PCE & S-PCE & A-PCE & S-PCE \\
\hline & \multicolumn{6}{|c|}{ TD (Gy) } \\
\hline $5 \%$ & $-*$ & 32.7 & $-*$ & 32.4 & 9.0 & 39.9 \\
\hline $10 \%$ & 4.3 & 38.3 & 1.1 & 37.7 & 16.1 & 43.8 \\
\hline $25 \%$ & 19.4 & 46.5 & 16.9 & 46.6 & 27.9 & 50.4 \\
\hline \multirow[t]{2}{*}{$50 \%$} & 34.4 & 54.7 & 34.3 & 56.5 & 41.0 & 57.7 \\
\hline & \multicolumn{6}{|c|}{ NTCP (probability) } \\
\hline 10 Gy & 0.00 & 0.00 & 0.17 & 0.00 & 0.06 & 0.00 \\
\hline 20 Gy & 0.26 & 0.01 & 0.29 & 0.01 & 0.14 & 0.00 \\
\hline 30 Gy & 0.42 & 0.04 & 0.43 & 0.04 & 0.29 & 0.01 \\
\hline 40 Gy & 0.60 & 0.12 & 0.59 & 0.13 & 0.48 & 0.05 \\
\hline 50 Gy & 0.76 & 0.35 & 0.73 & 0.33 & 0.68 & 0.24 \\
\hline
\end{tabular}

The upper row presents estimated $\mathrm{TD}_{5}, 10,25$ and $\mathrm{TD}_{50}$ according to the calculated model and grade of PCE.

The bottom row presents the NTCP for each $10 \mathrm{~Gy}$.

Regarding the LKB model, 10 Gy means gEUD whereas for the MHD-based model, 10 Gy means MHD. ${ }^{*} \mathrm{TD}_{5}$ for the MHD-based model was not achieved because it was outside the fitting curve. 
A-PCE: pericardial effusion in any grade; gEUD: generalised equivalent uniform dose; LKB:

Lyman-Kutcher-Burman; MHD: mean heart dose; MPD-based: mean pericardial dose-based; NTCP: normal tissue complication probability; S-PCE: symptomatic pericardial effusion; TD: $\mathrm{TD}_{50}$ is the dose that corresponds to a $50 \%$ risk of complications when the whole organ is irradiated. 

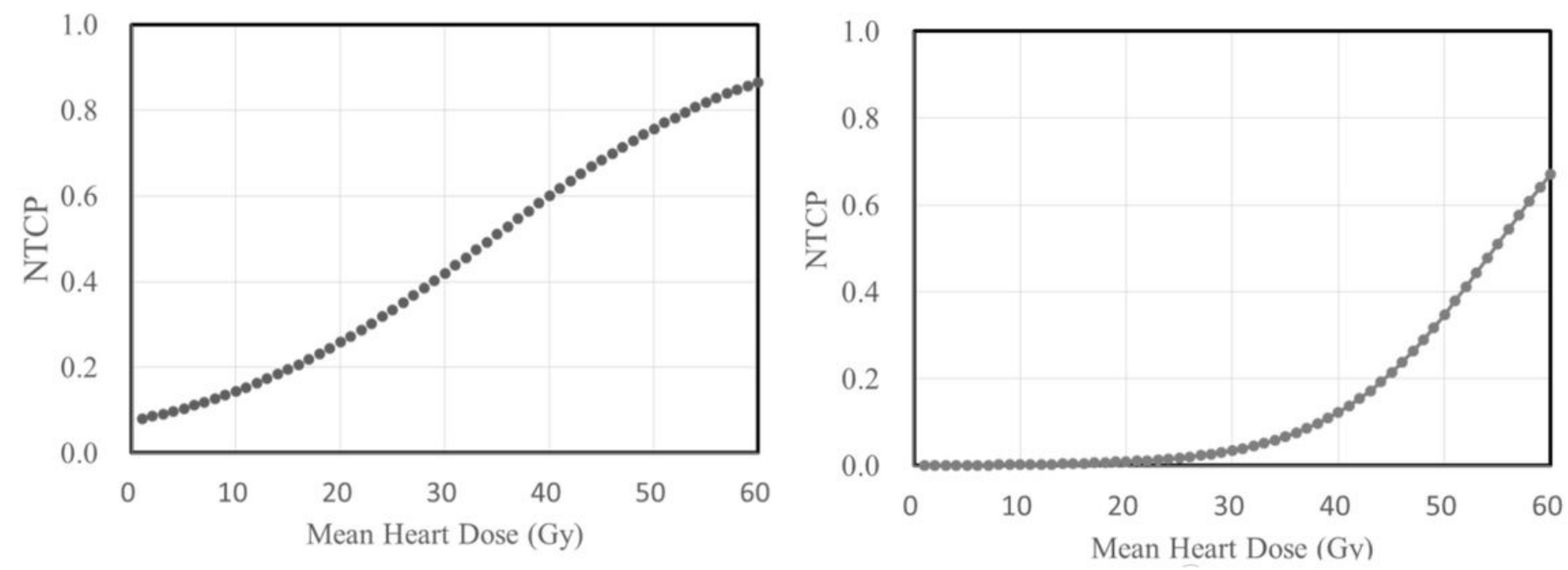

Figure 1

NTCP curves for A-PCE (a) and S-PCE (b). The NTCP curves were calculated by the logistic regression analysis derived from MHD. A-PCE, pericardial effusion in any grade; NTCP, normal tissue complicated probability; S-PCE, symptomatic pericardial effusion; MHD, mean heart dose
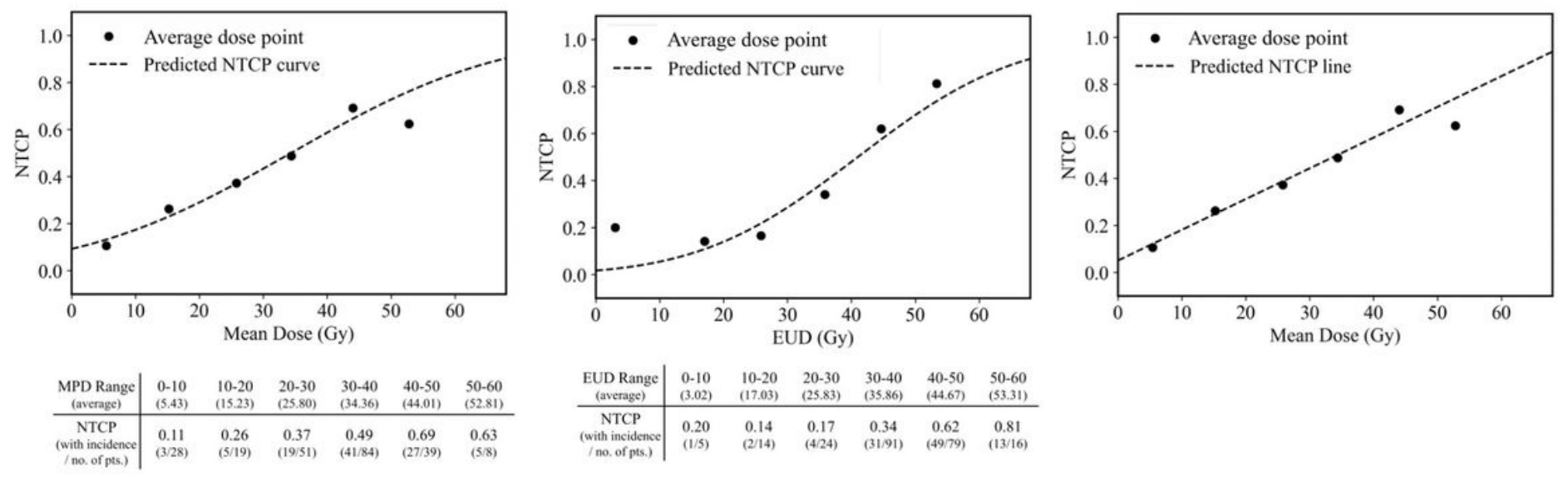

Figure 2

NTCP curves for A-PCE calculated by the MHD-based model (a and c) and LKB model (b). The x-axis indicates the MHD, which corresponds to the mean dose in the MHD-based model. Filled circles indicate average dose points for gEUD at 10 Gy intervals. NTCP curve by the MHD-based model also showed preferable fitting by linear regression (c). A-PCE, pericardial effusion in any grade; LKB model, LymanKutcher-Burman model; MHD, mean heart dose; NTCP, normal tissue complicated probability; No. of Pts, number of patients; gEUD, generalised equivalent uniform dose 

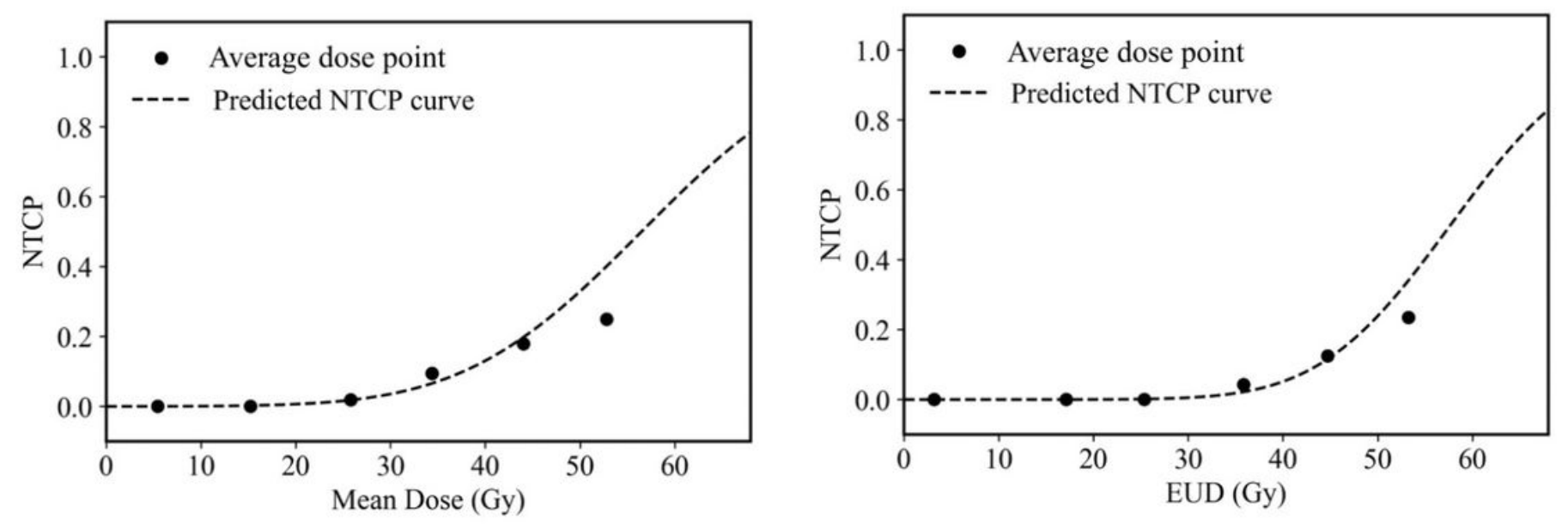

\begin{tabular}{c|cccccc}
$\begin{array}{c}\text { MPD Range } \\
\text { (average) }\end{array}$ & $\begin{array}{c}0-10 \\
(5.43)\end{array}$ & $\begin{array}{c}10-20 \\
(15.23)\end{array}$ & $\begin{array}{c}20-30 \\
(25.80)\end{array}$ & $\begin{array}{c}30-40 \\
(34.36)\end{array}$ & $\begin{array}{c}40-50 \\
(44.01)\end{array}$ & $\begin{array}{c}50-60 \\
(52.81)\end{array}$ \\
\hline $\begin{array}{c}\text { NTCP } \\
\text { (with incidence }\end{array}$ & $\begin{array}{c}0.00 \\
(0 / 28)\end{array}$ & $\begin{array}{c}0.00 \\
(0 / 19)\end{array}$ & $\begin{array}{c}0.02 \\
(1 / 51)\end{array}$ & $\begin{array}{c}0.10 \\
(8 / 48)\end{array}$ & $\begin{array}{c}0.18 \\
(7 / 39)\end{array}$ & $\begin{array}{c}0.25 \\
(2 / 8)\end{array}$
\end{tabular}

\begin{tabular}{c|cccccc}
$\begin{array}{c}\text { EUD Range } \\
\text { (average) }\end{array}$ & $\begin{array}{c}0-10 \\
(3.16)\end{array}$ & $\begin{array}{c}10-20 \\
(17.13)\end{array}$ & $\begin{array}{c}20-30 \\
(25.34)\end{array}$ & $\begin{array}{c}30-40 \\
(35.86)\end{array}$ & $\begin{array}{c}40-50 \\
(44.71)\end{array}$ & $\begin{array}{c}50-60 \\
(53.22)\end{array}$ \\
\hline $\begin{array}{c}\text { NTCP } \\
\text { (with incidence }\end{array}$ & $\begin{array}{c}0.00 \\
(0 / 5)\end{array}$ & $\begin{array}{c}0.00 \\
(0 / 12)\end{array}$ & $\begin{array}{c}0.00 \\
(0 / 23)\end{array}$ & $\begin{array}{c}0.04 \\
(4 / 92)\end{array}$ & $\begin{array}{c}0.13 \\
(10 / 80)\end{array}$ & $\begin{array}{c}0.24 \\
(4 / 17)\end{array}$
\end{tabular}

\section{Figure 3}

NTCP curves for S-PCE calculated by the (a) MHD-based model and (b) LKB model. The x-axis indicates gEUD, which corresponds to the mean dose in the MHD-based model. Filled circles indicate average dose points for gEUD at $10 \mathrm{~Gy}$ intervals. gEUD, generalised equivalent uniform dose; LKB model, LymanKutcher-Burman model; No. of Pts, number of patients; NTCP, normal tissue complicated probability; S$\mathrm{PCE}$, symptomatic pericardial effusion; MHD, mean heart dose

\section{Supplementary Files}

This is a list of supplementary files associated with this preprint. Click to download.

- supplfinal.docx 